\title{
Einstein's theory of theories and types of theoretical explanation
}

\author{
FRANCISCO FLORES
}

Department of Philosophy, Queen's University, Canada

\begin{abstract}
In this paper I draw on Einstein's distinction between "principle" and "constructive" theories to isolate two levels of physical theory that can be found in both classical and (special) relativistic physics. I then argue that when we focus on theoretical explanations in physics, i.e. explanations of physical laws, the two leading views on explanation, Salmon's "bottom-up" view and Kitcher's "top-down" view, accurately describe theoretical explanations for a given level of theory. I arrive at this conclusion through an analysis of explanations of mass-energy equivalence in special relativity.
\end{abstract}

\section{Introduction}

Part of Newton's legacy to philosophy is a distinction between two levels of physical theory. In his own work, Newton explicitly separates the framework of principles that govern all possible forces (i.e. the laws of motion of Principia and their corollaries), from theories that describe specific physical interactions within this framework, like his theory of universal gravitation (UG). Despite its Newtonian origins, this kind of distinction is not confined to so-called "classical" physics. In the context of special relativity (SR), one can also separate the collection of general physical principles from theories that describe interactions within this framework (like relativistic electrodynamics). In both cases, the general principles establish constraints that must be satisfied by other theories. In this paper, I draw on Einstein's distinction between "principle" and "constructive" theories to isolate this distinction between two levels of theories, which I refer to as "framework theories" and "interaction theories". I then argue that we do well to keep this distinction in mind when we address questions regarding the nature of theoretical explanations (i.e. explanations of laws) in physics. Using the explanation of mass-energy equivalence as an example, I propose that the distinction between "top-down" explanations (TDEs) and "bottom-up" explanations (BUEs) is really a symptom of the distinction framework and interaction theories.

$I$ begin, in Section 2, with an overview of Einstein's distinction between principle and constructive theories. In Section 3, I argue that a closer look at why Newton's theory of UG does not qualify as either a principle or a constructive theory 
suggests that the valuable core of Einstein's distinction is that it separates theories according to how they function in scientific theorizing. The "framework" vs. "interaction" theory distinction is introduced to isolate two levels of physical theory by preserving the core of Einstein's distinction and dispensing with its ontological and epistemological dimensions. I then argue, in Section 4, that there are clear examples of laws that receive only BUEs while others receive only TDEs. Einstein's mass-energy equivalence, which first receives what appears to be a BUE, is best regarded as having only a TDE. This, I propose, is because $E=m c^{2}$ is a law that belongs to a framework theory. I conclude with brief remarks suggesting that the framework vs. interaction theory distinction can help us understand why each view of scientific explanation faces the challenges it does, and why they seem successful where they do.

\section{Einstein's principle vs. constructive theory distinction}

Einstein's view of the structure of scientific theories is found, either explicitly or implicitly, in a variety of his popular and scientific papers. Throughout, Einstein maintains a distinction between theoretical physics and phenomenological physics (for example, see Einstein (1936)). The former consists of theories that are best understood as deductive structures whose starting points tend to become more removed from the world of everyday experience as science marches on. The virtues of theoretical physics include the logical unity of the foundations of its theories, and their simplicity. Phenomenological physics, on the other hand, "makes as much use as possible of concepts which are close to experience but, for this reason, has to give up, to a large extent, unity in the foundations" (1936, p. 302). Early theories of heat, electricity, and light are some of Einstein's favourite examples. In these theories,

heat, electricity and light are described by separate variables of state and material constants other than the mechanical quantities; and to determine all of these variables in their mutual temporal dependence was a task which, in the main, could only be solved empirically. (Einstein, 1936, p. 302)

Unlike the theories of theoretical physics, phenomenological theories do not begin from a small collection of axioms and definitions of concepts from which a variety of relationships between physical quantities can be derived. Instead the relations between the variables of state are arrived at, and justified, only empirically. For example, one finds the relationship between the temperature and pressure of a gas, or the relationship between the magnitude of an electric current and the intensity of the induced magnetic field, empirically instead of deducing these relationships theoretically. ${ }^{1}$ For Einstein,

as long as no principles are found on which to base the deduction [of existing or new phenomena], the individual empirical fact is of no use to the theorist; indeed he cannot even do anything with isolated general laws abstracted from experience. He will remain helpless in the face of separate results of empirical research, until principles which he can make the basis of deductive reasoning have revealed themselves to him. (Einstein, 1914, p. 221)

Thus, phenomenological physics is, first and foremost, a purely descriptive science that uses concepts which are ready to hand and that generates finished products, i.e. relationships between quantities with a paucity of consequences. Furthermore, for Einstein, there is a trend in the history of physics toward the theoretical deduction of 

within the theory, typically involves a process of idealization that takes us beyond our current, and past, observations. Einstein describes this process as "raising" an empirical generalization "to the status of a postulate" (1905a, p. 38).

A clear example of how conjectures, which have been corroborated by experiments, can be "raised to the status of postulate" appears in the introduction to Einstein's (1905a) paper on SR. Einstein first describes the results of then recent experiments in the following way: "as has already been shown to the first order of small quantities, the same laws of electrodynamics and optics will be valid for all frames of reference for which the equations of mechanics hold good" (1905a, p. 38). Einstein then says, "we will raise this conjecture (the purport of which will hereafter be called the "Principle of Relativity') to the status of a postulate" (1905a, p. 38). The conjecture is the claim that the experiments seek to establish, namely that to the first order of small quantities, the same laws of electrodynamics and optics are valid for all inertial frames of reference. To raise this to a postulate requires that we make an idealization, which takes us well beyond the first order of small quantities, and postulate the principle of relativity simpliciter. Einstein gives precisely this kind of description of how we discover physical principles - the starting points of principle theories-when he discusses Galileo's law of inertia in his popular book The Evolution of Physics (Einstein \& Infeld, 1961, pp. 5 ff.). There, Einstein argues that no amount of experiment will reveal that a body continues in a state of uniform motion when it is free from external forces. When we "raise this conjecture to the status of a postulate", we idealize the conditions of our experiment, in this case using a thought experiment, and postulate Galileo's law of inertia.

Einstein does not say very much about the epistemological status of the starting points of constructive theories. He does state that, unlike the postulates of a principle theory, the "hypothetical constituents" which are the starting points of constructive theories are not empirically discovered. Implicit in his remarks is the claim that one is free to postulate both the existence of these "hypothetical" elements and their properties. They are what Einstein might refer to as "free creations of the human mind", at least when they are first postulated. Of course, this does not prevent us from eventually having sufficient grounds to believe in their existence, nor does it commit us to anti-realism (or instrumentalism) regarding these "hypothetical" elements. For Einstein, what is remarkable about the kind of scientific creativity involved in postulating starting points of constructive theories is precisely that successful theories show that these "hypothetical constituents" do correspond to objective parts of physical systems. So, for example, although molecules begin as hypothetical constituents in the KTG, the empirical success of experiments to determine Avogadro's number, and of experiments to determine the size of molecules suggests that molecules are real constituents of matter.

The third way in which Einstein's distinction separates theories is according to their function: principle and constructive theories play different conceptual roles in scientific theorizing. For Einstein, the principles that lie at the foundations of a principle theory "give rise to mathematically formulated criteria which the separate processes or the theoretical representations of them have to satisfy" (1919a, p. 228). From our observations of physical systems, we glean the general characteristics that we believe all systems must satisfy. For any one of these conjectures, which are typically expressed as mathematical criteria, raising it to the level of a postulate means that we now regard it as a condition that any description of a physical system must satisfy. To say such a description must satisfy such a postulate means that if we encounter a case where the postulate appears to be violated, we first look for errors in our description of 
the physical system which might account for why the principle appears to be violated. We do not, in other words, begin by questioning the truth of the principle. For example, consider how this might work in the case of the principle of conservation of energy. First, we observe that the total energy in physical processes is conserved (within some margin of error). We then raise this conjecture to the level of a postulate. This means that if we encounter a physical system where energy is apparently not conserved, the missing energy must be found "somewhere".

As a more concrete example of how principles, such as the principle of conservation of energy, give rise to mathematical criteria that must be satisfied by the theoretical descriptions of physical processes supplied by constructive theories, consider the description of gases according to the KTG. In this constructive theory, molecules can be treated as tiny, rigid spheres. Newton's first law (a "principle" of Newtonian mechanics) restricts the allowable motion of the molecules so that, between collisions, the molecules must travel in straight lines with uniform velocities. Other restrictions may be imposed not by the laws of motion themselves, but by some of their consequences, such as the principle of conservation of momentum. According to the latter, if two molecules of equal mass, travelling with equal speeds, collide, the speed of the particles after the collision will be equal to the speed of the particles before the collision. Thus, in a principle theory, one invokes the postulates of the theory (in this case Newton's three laws of motion), along with the definitions of physical terms, and "seeks by analytical means to deduce necessary conditions, which the separate events [described by a constructive theory] have to satisfy" (Einstein, 1919a, p. 228).

Einstein does not treat the ontological, epistemological, and functional aspects of his distinction equally. Instead, he regards the ontological aspect of the distinction as primary - this is what really separates principle and constructive theories. The epistemological and functional aspects of the distinction are merely induced. The former is "induced" because Einstein believes he is just describing the epistemological status of the starting points of principle and constructive theories. In Einstein's characterization, there are no theories that begin with general, physical principles which are merely hypothetical postulates or "free creations of the human mind", because this is not how we come to know physical principles. Einstein explains, "the elements which form their basis and starting-points [i.e. the starting-points of principle theories] are not hypothetically constructed but empirically discovered ones" (Einstein, 1919a, p. 228). Similarly, there are no constructive theories that begin with "elements" that are empirically discovered; the starting points of constructive theories are always hypothetical constituents. Finally, since principle theories have "general characteristics of natural processes" as their starting points, these theories always establish the conditions that must be satisfied by the "hypothetical elements" of constructive theories. Thus, for Einstein, the functional dimension of the principle vs. constructive theory distinction is also induced by the ontological dimension.

\section{The framework vs. interaction theory distinction}

Einstein's distinction was arguably never meant to be exhaustive. And it is not difficult to find examples of theories that seem not to fall into either category; Newton's theory of UG is one such example. In this section, I will argue that investigating why UG cannot be classified as either a principle or a constructive theory demonstrates that the valuable core of Einstein's distinction is its functional dimension. By focusing on this 
aspect of the distinction, instead of focusing on the ontological aspect as Einstein does, we obtain a more robust distinction between what I call "framework" and "interaction" theories.

To determine whether a given theory is a principle or a constructive theory we cannot simply look at whether it contains a law that describes a "general characteristic of natural processes" or whether it postulates "hypothetical constituents". For if we do that we will trivialize Einstein's distinction as there will be cases where it will be equally plausible to characterize a given theory as a principle theory and as a constructive theory. The isolated fact that a theory has a "main law" with which we identify it, say the law of UG or the law of the electrostatic force, does not make it a principle theory any more than the fact that a theory postulates "hypothetical constituents" makes it a constructive theory. In either case, what is of utmost importance to Einstein's distinction is that the "main laws" or the "hypothetical constituents" of a theory are its "starting points". Without this, Einstein's distinction would lose its bite. But what, according to Einstein, does it mean to say that a physical law or certain "hypothetical constituents" are the "starting points" of a theory? Let me consider each type of theory in turn and thereby demonstrate that UG is neither a principle nor a constructive theory according to Einstein's criteria.

According to Einstein, a given physical law is a "principle" of a theory, i.e. it is a "starting point" of a principle theory, only if it is regarded as logically primary in the deduction of other laws. Einstein often compares a physical theory, by which he implicitly means a principle theory, to a deductive structure. In this analogy, the laws that are analogous to the axioms of a formalized theory are the "principles" of the theory. Thus, just as we cannot ask of a proposition in isolation whether it is an axiom or a theorem, the question concerning whether a given physical law is a "principle" of a theory cannot be asked of a law in isolation. In the context of Newtonian mechanics, Einstein regards the three laws of motion as the "axioms" of the theory from which "everything [beyond this] follows from the development of appropriate mathematical methods by means of deduction" (Einstein, Autobiographical Notes, p. 19). Thus, for Einstein it must be the case that the law of UG is not a "principle" because it is a consequence of the three laws of motion. ${ }^{5}$

While this rules out the possibility that UG is by itself a principle theory, it suggests that UG is part of a principle theory. After all, the principle theory is composed not just of its "starting points" but also contains all of their consequences. So it would seem that at least for Einstein, UG is part of the principle theory of Newtonian mechanics. However, it is only a very weak sense that we can say that UG is a consequence of Newton's three laws. Strictly speaking the law of UG cannot be derived from the three laws alone. This is because from the three laws we can only derive very general relationships concerning a variety of physical configurations. For example, Newton demonstrates how one can obtain a generalized version of Kepler's Harmonic Law in Corollary VI to Proposition IV in Book I of Principia. ${ }^{6}$ While this corollary plays a central role in Newton's argument for UG, we cannot derive any specific force law from the three laws of motion alone. We have to appeal to the phenomena, as Newton does in his argument for UG, if we want to determine the specific nature of a particular force. Thus, UG is not part of the principle theory of Newtonian mechanics either.

To determine whether UG is a constructive theory we have to consider whether the "hypothetical constituent" postulated by UG, namely the force of gravity, is its "starting point". In contrast to his characterization of principle theories, where the analogy to a "deductive structure" yields a fairly concrete sense to "starting points", in the case 
of constructive theories Einstein is not very clear about what it means for certain "hypothetical constituents" to be the "starting points" of a theory. We can, nevertheless, glean a necessary condition for the "starting points" of a constructive theory from Einstein's solitary example of this type of theory (namely the KTG): a "hypothetical entity" postulated by a theory is a "starting point" of that theory only if it is an explanatory primitive. Whatever physical laws the theory explains it does so on the basis of considering how the "hypothetical constituents" behave collectively.

If we ignore this necessary condition on what constitutes the "starting points" of a constructive theory, it seems UG is a constructive theory after all. One might reach this conclusion in the following way: UG is a constructive theory because for a given motion of a body under the influence of gravity, we must take account of all the separate gravitational forces acting on the body and combine them according to a "simple formal scheme", namely vector addition. We "construct" the complex behaviour we are trying to describe, say the path of a comet, by taking into account the collective "behaviour" of all the gravitational forces that act on the body. Thus, on this reading, and allowing that the notion of "hypothetical constituents" might include "theoretical entities" such as the gravitational force, UG comes out as a constructive theory.

But notice that the collective behaviour of gravitational forces cannot explain the law of UG in the way that the collective behaviour of molecules explains the ideal gas law. This is because each "component" gravitational force can only be determined by the very law in question. Indeed according to Einstein in Newton's hands gravitation remains unexplained: "his law of gravitational interaction is not supposed to be a final explanation but a rule derived by induction from experience" (Einstein, 1954, p. 258). So even if we grant that the force of gravity can be regarded as a "hypothetical constituent" used by the UG, it does not follow that UG is a constructive theory because the force of gravity is not a "starting point" of this theory in Einstein's sense. Thus, when we focus, as Einstein does, on the ontological aspect of the principle vs. constructive theory distinction (i.e. on what the "starting points" of a theory are), we find that some theories fall in neither category.

We can remedy this situation by focusing on the functional dimension of Einstein's distinction instead. At one end of a spectrum, we find theories that impose restrictions on other theories. The main elements of these "upper-level" theories are general physical principles (typically expressed as "laws") and definitions of physical terms which are expected to be applicable in the analysis of any physical system. Because these theories provide the scaffolding upon which other theories are built, I call them "framework theories". At the other end, there are theories that describe specific physical processes within the constraints imposed by the principles (or one of the consequences) of a framework theory. ${ }^{7}$ Since these theories routinely describe what physicists commonly refer to as "interactions", I refer to them as "interaction theories". The relationship between the framework vs. interaction theory distinction and Einstein's distinction is straightforward: all principle theories are framework theories and vice versa; all constructive theories are interaction theories but not vice versa. Thus, Einstein's distinction is a "special case" of the distinction I have introduced.

In the context of classical physics, the framework vs. interaction theory distinction allows us to separate the KTG and UG, on the one hand, from Newtonian mechanics (i.e. what is contained in Book I of Principia) on the other. Also, any theory that describes a specific force law can now be characterized as an interaction theory. This is because our speculations regarding the exact nature of this force (i.e. how it depends, 

establishes a general result regarding the relationships between the period and radius of an orbit, and the centripetal force required to maintain a particle in that orbit. The argument he provides, furthermore, is a derivation from his three laws. It seems Kitcher would agree that Newton's derivation of general "laws" like Proposition IV can be easily shown to be an explanation according to his view; it is a TDE because this "law" is no longer a "disconnected" fact we have to accept as brute. Instead, it is "unified" with a variety of other laws, like the Newtonian equivalence principle and the Galilean principle of relativity (both are corollaries to the laws of motion), which are also consequences of Newton's three laws. Together, these examples show that one can distinguish two types of theoretical explanations because the salient features of these explanations are quite different.

A question that seems natural at this stage is: why do these laws receive just this type of explanation? An instructive example for answering both of these questions is the explanation of Einstein's famous mass-energy equivalence, $E=m c^{2}$. There are two main ways to arrive at this result in SR. First, one can derive the equivalence of mass-energy, as Einstein (1905b) does the first time he proves this result, using a thought experiment that appeals to Maxwell's theory of electromagnetism. ${ }^{8}$ Einstein considers a body that emits two pulses of electromagnetic radiation (say, in the form of light) of equal energy in opposite directions with respect to a given coordinate system. By invoking a variety of physical principles, including the principle of relativity, and a transformation equation for the components of the electromagnetic field to analyse the act of emission from another inertial coordinate system, ${ }^{9}$ Einstein arrives at his celebrated result: "If a body gives off the energy $L$ in the form of radiation, its mass diminishes by $L / c^{2 "}(1905 \mathrm{~b}$, p. 71). Prima facie, this derivation of $E=m c^{2}$ seems particularly amenable to the description of scientific explanation favoured by proponents of the "bottom-up" approach. We have, it seems, identified a causal process that is responsible for the regularity we observe. Beyond predictive and descriptive knowledge, it seems we have the kind of knowledge about the "inner workings of nature" that justifies calling this a BUE. Even if our philosophical analysis of physical causation does not lead to the conclusion that the emission of energy caused the decrease in the mass of the body, we may still suppose that physics will eventually deliver the causal explanation we expect on philosophical grounds. We might argue, in other words, that although this may not be the best BUE available, it strongly suggests that this law will eventually receive this type of explanation. However, the history of physics proceeds in precisely the opposite direction.

The distinctive feature of the second way of deriving the equivalence of mass-energy is that these derivations do not appeal to any theory that describes a specific physical interaction, like Maxwell's theory. Historically, this approach appears shortly after Einstein's first derivation in the work of a variety of physicists, including Einstein himself, and culminates in the work of Ehlers et al. (1965). Unlike Einstein's first derivation, these derivations of $E=m c^{2}$ use not just the kinematical framework of SR but also the "new" dynamical quantities, relativistic momentum and relativistic kinetic energy. In Einstein's (1935) derivation, more than half the paper is devoted to arriving at the mathematical expressions that define these quantities. With the definitions of these quantities in hand, Einstein then analyses an inelastic collision between two particles, again invoking a variety of physical principles, to arrive at the desired result. ${ }^{10}$

Einstein's second derivation, and subsequent derivations, come much closer to being TDEs. In these derivations, the equivalence of mass and energy is arrived at by deriving it directly from other physical principles (which are taken as primitive) and the 
definitions of the new dynamical quantities. If we grant that this derivation unifies our knowledge, at least in so far as it is obtained from physical principles that can be used to derive a variety of other laws (like the velocity addition law of SR), then we have all the requirements of a TDE. So it seems we have good grounds for adopting the conclusion that the same law is receiving two different kinds of explanation. However, to do so would be a mistake.

To see why this would be a mistake, we have to recognize that the distinction between the two types of theoretical explanation is really a manifestation of the distinction between framework and interaction theories. Interaction theories, and Einstein's constructive theories, are precisely the kind of theories that yield BUEs. Beyond purely descriptive and predictive knowledge, this type of theory postulates underlying causal mechanisms for the laws we wish to explain. Thus, interaction theories (and constructive theories) yield BUEs. On the other hand, framework theories are restricted to offering TDEs. This is because from the physical principles that constitute a framework theory one can only derive other laws which must be satisfied by all physical processes. A principle theory can never offer a detailed description of a chain of events in the way that an interaction theory, and particularly a constructive theory, can. If this is correct, then we can begin to understand why $P V=n R T$ receives a BUE from the KTG while, for example, the Galilean relativity principle in Newtonian mechanics receives a TDE. How can this illuminate my earlier claim that it is a mistake to regard $E=m c^{2}$ as having both types of explanations?

In his first derivation, Einstein explains the equivalence of mass-energy by appealing to an interaction theory, namely Maxwell's theory of electromagnetism. The framework theory, in this case, is the kinematical part of SR. Physicists often remark that Einstein's original derivation is undesirable precisely because it depends on Maxwell's theory. Einstein himself expresses this sentiment in the opening paragraph of the article that contains his second derivation:

The question as to the independence [from Maxwell's theory] of those relations [like $E=m c^{2}$ ] is a natural one because the Lorentz transformation, the real basis of the special theory, in itself has nothing to do with the Maxwell theory and because we do not know the extent to which the energy concepts of the Maxwell theory can be maintained in the face of the data of molecular physics. (Einstein, 1935, p. 223)

The methodological prescription implicit in these remarks is that whenever one has a result that applies to any physical process whatsoever, one should look for a derivation at the level of the framework theory, i.e. one should look for a TDE. As Einstein explains, this is important because a general result like $E=m c^{2}$ should not depend on the correctness of an interaction theory like Maxwell's theory. In this case, this is particularly pressing because of experimental results showing that energy is quantized. Thus, we have good grounds for rejecting Einstein's first derivation as an explanation, especially when we consider that despite appearances to the contrary, this derivation does not reveal the causal mechanisms responsible for the equivalence of mass-energy after all. Thus, the closest thing we have to an explanation of $E=m c^{2}$ is a TDE.

\section{Conclusion}

Once we recognize that the type of theoretical explanation a law receives depends on whether it is part of a framework or interaction theory, we can understand why 
proponents of either the "bottom-up" or "top-down" approach select the examples they do. For example, it should come as no surprise now that Salmon's paradigm of a theoretical explanation is the KTG's explanation of the ideal gas law. Furthermore, we can also see why the respective views of explanation work so well where they do. For example, one of the challenges for the "top-down" view is to account for the problem of asymmetry. But once we recognize that TDEs only occur at the level of the framework theory, asymmetry is no longer a problem. This is because at this level explanations are symmetric, which should come as no surprise since these explanations are non-causal. Where asymmetry is really necessary for an explanation is at the level of interaction theories where BUEs achieve it by their appeal to causes. Finally, if I am correct, then we can meet Salmon's challenge to characterize the relationship between BUEs and TDEs. While we cannot say that BUEs are "constrained" by TDEs, as we say of the two corresponding levels of theory, we can say that BUEs require fundamental laws. And these fundamental laws, which are inevitably part of framework theory, accept only TDEs.

\section{Acknowledgement}

I would like to thank Robert DiSalle, Bill Demopoulos, and Robert Clifton for their helpful comments. A portion of the research for this work was funded by the Social Sciences and Humanities Research Council of Canada.

\section{Notes}

1. This is not to say that empirical observations are not "theory laden". The point is that given a background of theoretical concepts, like temperature and pressure, in a phenomenological theory, we find the mathematical relationships that obtain between these quantities empirically.

2. The requirement that the starting points of a principle theory be "general characteristics of natural processes" (1919a, p. 228) precludes treating phenomenological laws as the starting points of a principle theory. Although, for Einstein, all physical principles (i.e. the starting points of principle theories) are empirical generalizations, the converse is, of course, not the case. For example, one cannot treat Kepler's laws as principles because they are not sufficiently general. As expressed by Kepler, these laws apply only to the planets that orbit the sun. Einstein explains: "The third law [Kepler's] cannot simply be transferred quantitatively to other central bodies than the sun (there is, e.g., no relation between the period of revolution of a planet round the sun and that of a moon round its planet)" (Einstein, 1954, p. 255).

3. In the KTG, the "hypothetical constituents" are the gas molecules. The "simple formal scheme" used by the KTG to account for the complex phenomena of gases, like Boyle's law and Maxwell's law, is to treat molecules as perfectly elastic, rigid spheres of very small, but finite, diameter and to analyse their collisions within the framework of Newtonian mechanics.

4. One need not take "matter" to mean physical bodies. One can also include fields, like the electromagnetic field, as is traditionally done in SR.

5. Notice how different this is from the case of the principle of the constancy of the velocity of light in the context of the special theory of relativity. This "law" is not derived from any other law, and no other laws serve to constrain it in the way that the law of UG is constrained by Newton's three laws.

6. Newton shows that regardless of the kind of force in question, if the period of the orbit is proportional to $R^{n}$ (where $R$ is the radius of the orbit), then the centripetal force acting on that body varies as $R^{-(2 n-1)}$.

7. Einstein's parallel claim concerning how constructive theories must satisfy the constraints imposed by a principle theory is a bit stronger than it has to be. Although our aim is to ensure that the descriptions given by interaction theories (or constructive theories) satisfy all the constraints imposed by the framework theory, there are cases where this cannot be done. However, in cases such as this, difficult choices have to be made. An option that can yield conceptual gain in these cases is to attempt to find a new framework theory. This, in outline, describes the relationship between Maxwell's theory of electromagnetism and the Newtonian framework theory, and the transition to SR. 
8. Although a number of authors, including Jammer (1961), have argued that this proof does not work, Torretti \& Stachel (1982) have more recently shown that this proof does not contain any errors. Still, like other proofs of this result, what cannot be established is that all the mass of the body can be convertible to energy. This must be accepted as an additional hypothesis in this proof.

9. Einstein must also use the law of conservation of momentum, the principle of conservation of energy, a transformation equation for the energy of an electromagnetic pulse, and the existence of a Newtonian limit in his derivation.

10. For a closer examination of Einstein's (1935) derivation see Flores (1998).

\section{References}

EARMan, J. (1989) World Enough and Spacetime (Cambridge, MA, MIT Press).

EHLERs, J., RNNDLER, W. \& PENROSE, R. (1965) Energy conservation as the basis of relativistic mechanics. II, American foumal of Physics, 35, pp. 995-997.

EINSTEN, A. (1905a) On the electrodynamics of moving bodies, in: A. EINSTEIN et al. (Eds) The Principle of Relativity (New York, Dover, 1952), pp. 35-65.

EINSTEIN, A. (1905b) Does the inertia of a body depend upon its energy-content?, in: A. EINSTEIN et al. (Eds) The Principle of Relativity (New York, Dover, 1952), pp. 69-71.

EInstern, A. (1914) Principles of theoretical physics, in: A. EInstern, Ideas and Opinions. Translated by S. BarmanN (New York, Bonanza Books, 1954), pp. 220-223.

EINSTEIN, A. (1919a) What is the theory of relativity, in: A. Einstern, Ideas and Opinions. Translated by S. BARMANN (New York, Bonanza Books, 1954), pp. 227-232.

Einstern, A. (1919b) Time, space, and gravitation, in: A. Ernstern, Out of My Later Years (New York, Philosophical Library, 1950), pp. 54-58.

EINSTEIN, A. (1935) Elementary derivation of the equivalence of mass and energy, American Mathematical Society Bulletin, 41, pp. 223-230.

Einstern, A. (1936) Physics and reality, in: A. EINSTEIN, Ideas and Opinions. Translated by S. Barmann (New York, Bonanza Books, 1954), pp. 290-323.

Einstern, A. (1950) Out of My Later Years (New York, Philosophical Library).

Einstern, A. (1954) Ideas and Opinions. Translated by S. BARMANN (New York, Bonanza Books).

Einstein, A. \& INFELd, L. (1961) The Evolution of Physics (New York, Simon and Schuster).

Einstein, A. et al. (1952) The Principle of Relativity (New York, Dover).

FLORES, F. (1998) Einstein's 1935 derivation of $E=m c^{2}$, Studies in the History and Philosophy of Moderm Physics, 29, pp. 223-243.

Jammer, M. (1961) Concepts of Mass in Classical and Modem Physics (Cambridge, MA, Harvard University Press).

KITCHER, P. (1989) Explanatory unification and the causal structure of the world, in: P. KITCHER \& W. Salmon (Eds) Minnesota Studies in the Philosophy of Science, Vol. XIII (Minnesota, University of Minnesota Press), pp. 410-503.

Newton, I. (1713) Mathematical Principles of Natural Philosophy. Translated by F. Cajoru (Berkeley, University of California Press, 1962).

RindLer, W. (1991) An Introduction to Special Relativity, 2nd edn (New York, Oxford University Press).

SAlmon, W. (1989) Four decades of scientific explanation, in: P. KrTcher \& W. SAlmon (Eds) Minnesota Studies in the Philosophy of Science, Vol. XIII (Minnesota, University of Minnesota Press), pp. 3-219.

TORRETTI, R. \& STACHEL, J. (1982) Einstein's first derivation of mass-energy equivalence, American fournal of Physics, 50, pp. 760-761.

\section{Note on contributor}

Francisco Flores obtained his PhD in Philosophy at the University of Western Ontario in June 1998. His thesis "Levels of Theory and Types of Theoretical Explanation" uses Einstein's distinction between "principle" and "constructive" theories to address questions regarding the nature of theoretical explanation in physics. His research interests include spacetime theories, history of analytic philosophy, and the philosophy of Kant. Correspondence: Department of Philosophy, Queen's University, Kingston, Canada K7L 3N6. E-mail: 8ff@post.queensu.ca 